\title{
Gastrointestinal tract aspergilloma: Possible cause of malabsorption
}

\author{
R J Prescott, N Y Haboubi, I E Burton
}

\begin{abstract}
A 62 year old man with chronic lymphocytic leukaemia presented with malabsorption, the cause of which could not be found during life. Necropsy examination showed aspergillosis, limited to the stomach, where tumour-like masses were seen, the oesophagus, and lungs. This case illustrates the problems of diagnosing fungal infections in life and the importance of clinicopathological correlation at necropsy.
\end{abstract}

$(\mathcal{C}$ Clin Pathol 1994;47:170-171)

Department of

Histopathology,

University Hospital of

South Manchester,

Nell Lane, West

Didsbury,

Manchester, M20 8LR

R J Prescott

N Y Haboubi

Department of

Haematology

I E Burton

Correspondence to:

Dr R J Prescott

Accepted for publication

29 July 1993

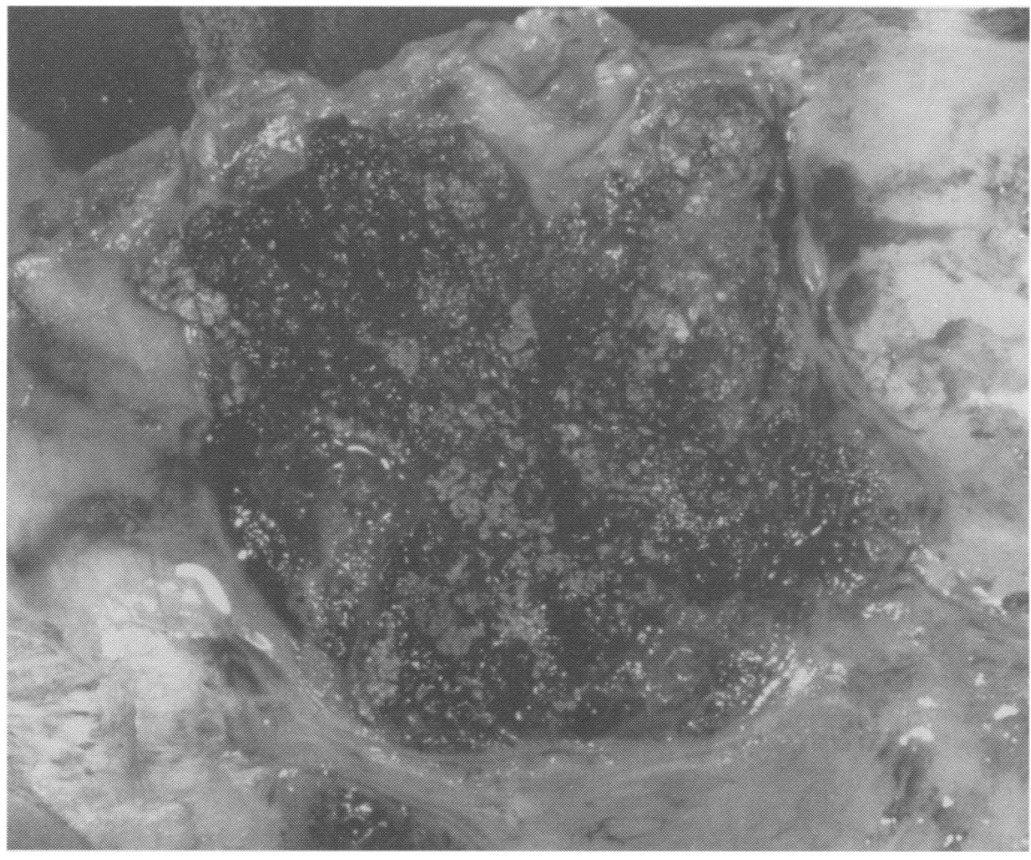

Figure 1 Gastric mass showing surface ulceration and haemorrhage.

A 62 year old white man presented with mild anaemia and a chest infection due to chronic lymphocytic leukaemia. He had a history of partial gastrectomy. Haematological results showed: haemogloblin $95 \mathrm{~g} / \mathrm{l}$, mean corpuscular volume (MCV) $108 \mathrm{fl}$, mean corpuscular haemoglobin $(\mathrm{MCH}) 36.7 \mathrm{pg}$, white cell count $69.9 \times 10^{9} / 1$ with lymphocytes $60.9 \times 10^{9} / 1$, an ESR $77 \mathrm{~mm} /$ first hour, vitamin B12 estimation $255 \mathrm{ng} / 1$ and serum folate $4 \cdot 1 \mu \mathrm{g} / 1$. A bone marrow biopsy specimen showed $80 \%$ infiltration with small lymphocytes. He improved after six courses of chlorambucil and prednisolone $(20 \mathrm{mg}$ twice a day). About a year later his haemoglobin fell to $83 \mathrm{~g} / 1$ with a normal white cell differential, platelets $128 \times 10^{9} / 1$, and reticulocyte count $5 \%$ associated with a positive antiglobulin test (anti-IgG only). Treatment with continuous high dose steroids and azathioprine for two months exacerbated a weight loss of $10 \mathrm{~kg}$, associated with diarrhoea with pronounced malabsorption (faecal fat excretion $38 \mathrm{mmol} /$ day, normal $<18 \mathrm{mmol} /$ day). A barium meal showed a partial gastrectomy and gastroenterostomy and no other abnormality.

A swinging fever was attributed to cholecystitis associated with an enlarged gallbladder. This partially responded to broad spectrum antibiotics. Gastric and duodenal biopsy specimens taken at gastroscopy and endoscopic retrograde cholangiopancreatography showed gastric metaplasia and normal duodenal mucosa. A chest $x$-ray picture, ultrasound and computed tomographic scanning of the abdomen were normal. Bacteriological studies including fungal cultures and $\mathrm{C}$ reactive protein were unhelpful in the diagnosis of fungal infection.

After a second gastroscopy he developed persistent hoarseness, then aphonia with continued anorexia, abdominal pain, and weight loss. His fever continued until death, in spite of intravenous treatment with amphotericin B and numerous antibiotics.

\section{Post mortem findings}

Post mortem examination showed extensive ulceration of the lower two-thirds of the oesophagus and several fungating tumour-like masses in the stomach, located in the cardia and body along the greater curvature. The largest mass measured $5 \times 5 \times 4 \mathrm{~cm}$ and in total they occupied about $40 \%$ of the gastric surface area (fig 1). The duodenum, small, and large intestines looked normal. The larynx looked normal, but both lungs showed macroscopic evidence of bronchopneumonia. There was no lymphadenopathy and the spleen weighed $295 \mathrm{~g}$ and was congested. The other organs looked normal. 
Figure 2 Dense eosinophilic precipitates outlining the hyphal walls, representing the SplendoreHoeppli phenomenon (haematoxylin and eosin).

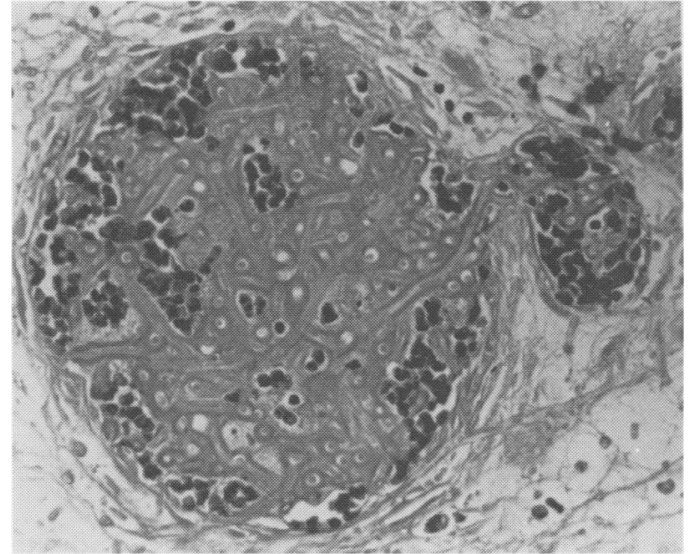

Microscopically, the oesophageal ulcers and gastric masses were composed of acute inflammatory exudate admixed with dichotomously branching, septate fungal hyphae typical of aspergillus. There was penetration of fungal elements into the oesophageal and gastric muscle coats and angio-invasion was a noticeable feature. Some of the hyphae within blood vessels had thickened eosinophilic outlines representing the Splendore-Hoeppli phenomenon (fig 2). The lungs contained foci of acute inflammation associated with aspergillus organisms. There was no evidence of fungal spread in any of the other organs. The spleen showed red pulp congestion only and there was no evidence of a leukaemic infiltrate in any of the sampled organs including bone marrow.

\section{Discussion}

The clinical presentation of aspergillosis is dominated by reports of lung, central nervous system, bone and paranasal sinus involvement. ${ }^{1}$ Only one report emphasises the high prevalence $(47 \%)$ of gastrointestinal disease, which is invariably associated with high dose steroids and lung disease. ${ }^{2}$ There are also a few cases of gastrointestinal haemorrhage associated with aspergillus invasion. Cohen and Heffner describe a patient with diarrhoea and acute myeloid leukaemia caused by distal ileal infarction, ${ }^{3}$ and Weingrad et al, a case with diarrhoea caused by bowel wall invasion. ${ }^{4}$ Vascular invasion with aspergillus has also been shown to produce bleeding from colonic ${ }^{5}$ and duodenal ulcers ${ }^{6}$ and perforation. ${ }^{2}$ Cappell reported invasion of the whole gastrointestinal tract with ulcers. ${ }^{7}$ Surprisingly, gastrointestinal aspergillosis is rarely seen in AIDS. ${ }^{8}$

In the absence of any other cause for diarrhoea producing malabsorption and weight loss in this patient, we presume that it was caused by gastrointestinal aspergillosis, in spite of negative cultures during life.

Another clinical clue which was missed in our patient was the development of persistent hoarseness, as described by Han et al, ${ }^{9}$ caused by fungal invasion of the larynx in immunosuppressed children.

In aspergillus infections the portal of entry is the lungs and, following angioinvasion, wide dissemination is possible, although in our case this was limited to the upper gastrointestinal tract.

Gross descriptions of gastrointestinal aspergillus infections include ulcers of varying configuration with or without perforation, infarction, and pseudomembranes, but tumour-like masses have not been described. $^{210}$ The microscopic features of aspergillus organisms are well described apart from the Splendore-Hoeppli phenomenon, an immunological reaction to fungal elements which are coated with immune complexes. ${ }^{11}$

There has been an increase in the number of post mortem cases of invasive aspergillosis in the past decade. ${ }^{1}$ Despite the increased clinical awareness, diagnosis of these infections during life remains at $25 \% .{ }^{12}$ The role of post mortem examinations and clinicopathological audit in studying the evolution of these infections cannot be over emphasised.

1 Boon AP, O'Brien D, Adams DH. 10 year review of invasive aspergillosis detected at necropsy. 7 Clin Pathol 1991;44:452-4.

2 Young PP, Bennett JE, Vagel CL, Carbone PP, Devita VJ. Aspergillosis: the spectrum of the disease in 98 patients. Med (Balt) 1970;49:147-73.

3 Cohen R, Heffner JE. Bowel infarction as the initial manifestation of disseminated aspergillosis. Chest 1992;101: festation

4 Weingrad DN, Knapper WH, Gold J, Mertelsmann G. Aspergillus peritonitis complicating perforated appendicitis in adult acute leukaemia. I Surg Oncol 1982; 19:5-8.

5 Kinder RB, Jourdan MH. Disseminated aspergillosis and bleeding colonic ulcers in renal transplant patients. f Roy Soc Med 1985;78:338-9.

6 Welsh RA, McClinton LR. Fatal gastrointestinal haemorrhage due to aspergillus infection. Arch Pathol 1954; 57:379-82.

7 Levitz SM. Aspergillosis. Infect Dis Clin North Am 1989; 3:1-18.

8 Cappel MS. Extensive gastrointestinal aspergillosis associated with AIDS. Dig Dis Sci 1991;36:1500-2.

9 Hass A, Hyatt AC, Kattan M, Weiner MA, Hodes DS. Hoarseness in immunocompromised children: association with invasive fungal infection. $\mathcal{F}$ Pediatr 1987; 111:731-3.

10 Smith JMB. Mycotic infections at autopsy. A comparative study of 162 cases. Am f Clin Pathol 1969;51:470-6.

11 Schwarz J. Aspergillosis. Annu Pathol 1973;8:81-107.

12 Myerowitz RL. The pathology of opportunistic infections with pathogenetic, diagnostic and clinical correlations. New pathogenetic, diagnostic 